\title{
ABDOMEN AGUDO DE ETIOLOGÍA INUSUAL: DIVERTÍCULO DE MECKEL PERFORADO POR UNA ESPINA DE PESCADO.
}

\author{
Cantos Gavilanes Iván René ${ }^{*}$, Vargas Córdova Ronnal Patricio², Vásconez \\ González Jorge Eduardo ${ }^{3}$, Mecías Córdova Rody Alejandro ${ }^{4}$, León Sanguano \\ Daysi Alejandra ${ }^{5}$, Yunga Atapuma Diego Ricardo ${ }^{4}$.
}

DOI: $10.48018 /$ rmv.v32.11.1

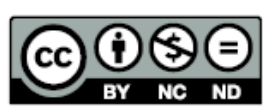

OPEN ACCESS

te artículo está bajo una licencia de Creative Commons de tipo Reconocimien- No comercial - Sin obras

1 Universidad Central del Ecuador. Facultad de Ciencias Médicas. Posgrado de Cirugía General y Laparoscópica. Quito, Ecuador.

2 Hospital General San Francisco IESS. Médico del Servicio de Cirugía Bariátrica - Endócrino Metabólica. Quito, Ecuador.

3 Hospital General San Francisco IESS. Médico en funciones hospitalarias. Quito, Ecuador.

4 Hospital General San Francisco IESS. Médico del Servicio de Cirugía General. Quito, Ecuador.

5 Pontificia Universidad Católica del Ecuador. Facultad de Medicina. Posgrado de Cirugía General y Laparoscópica. Quito, Ecuador.

ORCID ID:

Cantos Iván René

orcid.org/0000-0002-3664-5850.

Vargas Ronnal Patricio

orcid.org/0000-0001-5487-4000.

Vásconez Jorge Eduardo

orcid.org/0000-0003-4573-6217.

Mecías Rody Alejandro

orcid.org/0000-0003-2145-2877.

León Daysi Alejandra

orcid.org/0000-0002-6531-2350.

Yunga Diego Ricardo

orcid.org/0000-0003-2205-0693.

*Corresponding author: Cantos Gavilanes Iván E-mail: cantosgavilanesivan@gmail.com

Received: 01 - Nov - 2020

Accepted: 26 - Nov - 2020

Publish: 01 - Feb - 2021

CARE 2017 Check List statement: The authors have real the CARE 2017 Check List and the manuscript was prepared and revised according to the CARE 2017 Checklist.

Conflict of interest: All authors declared that there are no conflicts of interest.

Financial disclosure: The authors have no financial relationships relevant to this article to disclose.

Authors' contribution: RV, RM: Conceptualization of the project. DL, DY: information collection. IC, JV: manuscript writing. IC, RV: Critical review of the article. All the authors reviewed and approved the final manuscript.

Forma de citar este artículo: Cantos Gavilanes IR, Vargas Córdova RP, Vásconez González JE, Mecías Córdova RA, León Sanguano DA, Yunga Atapuma DR. ABDOMEN AGUDO DE ETIOLOGÍA INUSUAL: DIVERTÍCULO DE MECKEL PERFORADO POR UNA ESPINA DE PESCADO. Rev Med Vozandes. 2021; 32 (1):

\section{Resumen}

El divertículo de Meckel es la malformación congénita más común del tracto digestivo; tiene una prevalencia que va del $0.3 \%-2.9 \%$ en la población general de acuerdo con las diferentes publicaciones, su incidencia es mayor en el sexo masculino con una relación 1.5-4:1. En general, presentan un curso asintomático a lo largo de la vida, pero se estima que el $4,2 \%$ tendrán alguna complicación asociada, y el 3\% requerirán hospitalización y cirugía.

Paciente masculino de 37 años, quien acudió por cuadro de dolor abdominal inespecífico, al examen físico se evidenció un abdomen doloroso en mesogastrio y fosa iliaca derecha, sin signos de irritación peritoneal. Los estudios de laboratorio sin alteraciones; al no haber mejoría del cuadro abdominal, se complementó con estudio tomográfico computarizado, en el cual se evidenció una imagen lineal hiperdensa en íleon distal en relación con probable cuerpo extraño.

Se decidió resolución quirúrgica mediante abordaje laparoscópico; al no disponer de equipos de laparoscopía se realizó laparotomía infraumbilical.

Se identificó cuerpo extraño en relación con espina de pescado que atraviesa el divertículo de Meckel a $80 \mathrm{~cm}$ de la válvula ileocecal. Se realizó diverticulectomía con grapadora linear sin complicaciones, el histopatológico reportó divertículo de Meckel sin evidencia de tejido ectópico, y es dado de alta a las 48 horas.

Conclusiones: la presencia de un divertículo de Meckel como etiología de un abdomen agudo debe tenerse en cuenta y formar parte del diagnóstico diferencial del dolor en fosa iliaca derecha; siendo rara la perforación por cuerpo extraño.

Palabras clave: Divertículo de Meckel, abdomen agudo, cuerpos extraños, perforación intestinal.

\section{Abstract \\ ACUTE ABDOMEN OF UNUSUAL ETIOLOGY: PERFORATED MECKEL'S DIVERTICULUM BY A FISH BONE.}

Meckel's diverticulum is the most common congenital malformation of the digestive tract; It has a prevalence that ranges from $0.3 \%$ $2.9 \%$ in the general population according to different publications, its incidence is higher in males with a 1.5-4: 1 ratio.

In general, they present an asymptomatic course throughout life, but it is estimated that $4.2 \%$ will have some associated complication, and $3 \%$ will require hospitalization and surgery. 
Keywords: Meckel's Diverticulum, Foreign Bodies, Acute abdomen, Intestinal Perforation.
A 37-year-old male patient who presented with nonspecific abdominal pain, the physical examination revealed a painful abdomen in the mesogastrium and right lower quadrant, without signs of peritoneal irritation. Laboratory studies without alterations; as there was no improvement in the abdominal condition, it was complemented with a computed tomographic study, which showed a hyperdense linear image in the distal ileum in relation to a probable foreign body.

A surgical resolution was decided using a laparoscopic approach; as laparoscopy equipment was not available, an infraumbilical laparotomy was performed.

A foreign body was identified in relation to a fishbone that crosses Meckel's diverticulum at $80 \mathrm{~cm}$ from the ileocecal valve. A diverticulectomy with linear stapler was performed without complications, the histopathology reported Meckel's diverticulum without evidence of ectopic tissue, and he was discharged after 48 hours.

Conclusions: the presence of a Meckel diverticulum as an etiology of an acute abdomen should be taken into account and be part of the differential diagnosis of pain in the right lower quadrant; foreign body perforation being rare.

\section{Introducción}

El divertículo de Meckel (DM) se forma entre la sexta y décima semana de gestación debido a la obliteración incompleta del conducto onfalomesentérico (1,2); es considerada la malformación congénita más común del tracto digestivo(3), representando por sí sola el $90 \%$ de las patologías del conducto vitelino(1).

El DM es una estructura tubular ciega originada en un asa ileal, que se encuentra localizada en el borde anti mesentérico entre $7-200 \mathrm{~cm}$ (promedio $52.4 \mathrm{~cm}$ ) proximal a la válvula ileocecal; con una longitud y diámetro de $0.4-11 \mathrm{~cm}$ (promedio $3.05 \mathrm{~cm}$ ) y de $0.3-7 \mathrm{~cm}$ (promedio $1.58 \mathrm{~cm}$ ), respectivamente.

Por histología tiene todas las capas de la pared intestinal, en ocasiones su mucosa posee tejido ectópico, siendo el tejido gástrico el más frecuente $(4,6 \%-71 \%)$, seguido del tejido pancreático $(0-12 \%)^{(4,5)}$.

Su prevalencia es variable, según Hansen \& Søreide va del $0.3 \%$ $2.9 \%$ en la población general, pudiendo alcanzar hasta el $9 \%$, con una incidencia mayor en los hombres con una relación 1.5-4:1(5). En general, presentan un curso asintomático a lo largo de la vida, pero se estima que el $4,2 \%$ tendrán una complicación asociada a éste que necesitarán hospitalización y un 3\% requerirá cirugía(1).

Cuando un DM es sintomático sus manifestaciones clínicas son variables y en este contexto pueden presentarse asociadas a una complicación; siendo en su mayoría sangrado, obstrucción, inflamación y perforación ${ }^{(5)}$.

El sangrado es secundario a la presencia de tejido gástrico ectópico que genera ulceración; por otra parte, el proceso inflamatorio es menor al 1\%, pudiendo presentarse con un cuadro clínico severo si se asocia con perforación y peritonitis(2,3).
La obstrucción se presenta en el 14 al $40 \%$ de los casos, y se manifiesta por vólvulos, tumoraciones benignas o malignas y, también podría ser el resultado de la intususcepción del DM en el intestino ${ }^{(2,4,5)}$

Una complicación extremadamente rara es la perforación secundaria a inflamación, necrosis, obstrucción o una úlcera péptica y es aún más raro por cuerpos extraños como espina de pescado, palillo de dientes, hueso de pollo, monedas, baterías, etc.) ${ }^{(1-5)}$.

El objetivo del estudio es describir la prevalencia y el manejo quirúrgico del divertículo de Meckel perforado por cuerpo extraño. Este estudio sigue las directrices CARE(6) para el reporte de casos clínicos y el consentimiento informado ha sido obtenido para su publicación

\section{Descripción del caso}

Paciente masculino de 37 años, sin antecedentes patológicos, quien acudió al servicio de emergencia por cuadro de dolor abdominal inespecífico de 30 horas de evolución, al examen físico se evidenció un abdomen doloroso en mesogastrio y fosa iliaca derecha, sin signos de irritación peritoneal.

Exámenes complementarios sin alteración (leucocitos $6,54 \times 10^{3} \mathrm{UL}$, neutrófilos $3,47 \times 10^{3}$ 
$\mathrm{UL}$; $53 \%$, hemoglobina $16.9 \mathrm{~g} / \mathrm{dl}$, hematocrito $48.3 \%$ ); por persistencia del cuadro abdominal, se complementó con una tomografía computarizada (TC) contrastada de abdomen y pelvis, en la cual se evidencia imagen lineal hiperdensa en íleon distal en probable relación con cuerpo extraño, sin neumoperitoneo ni liquido libre (figura 1).

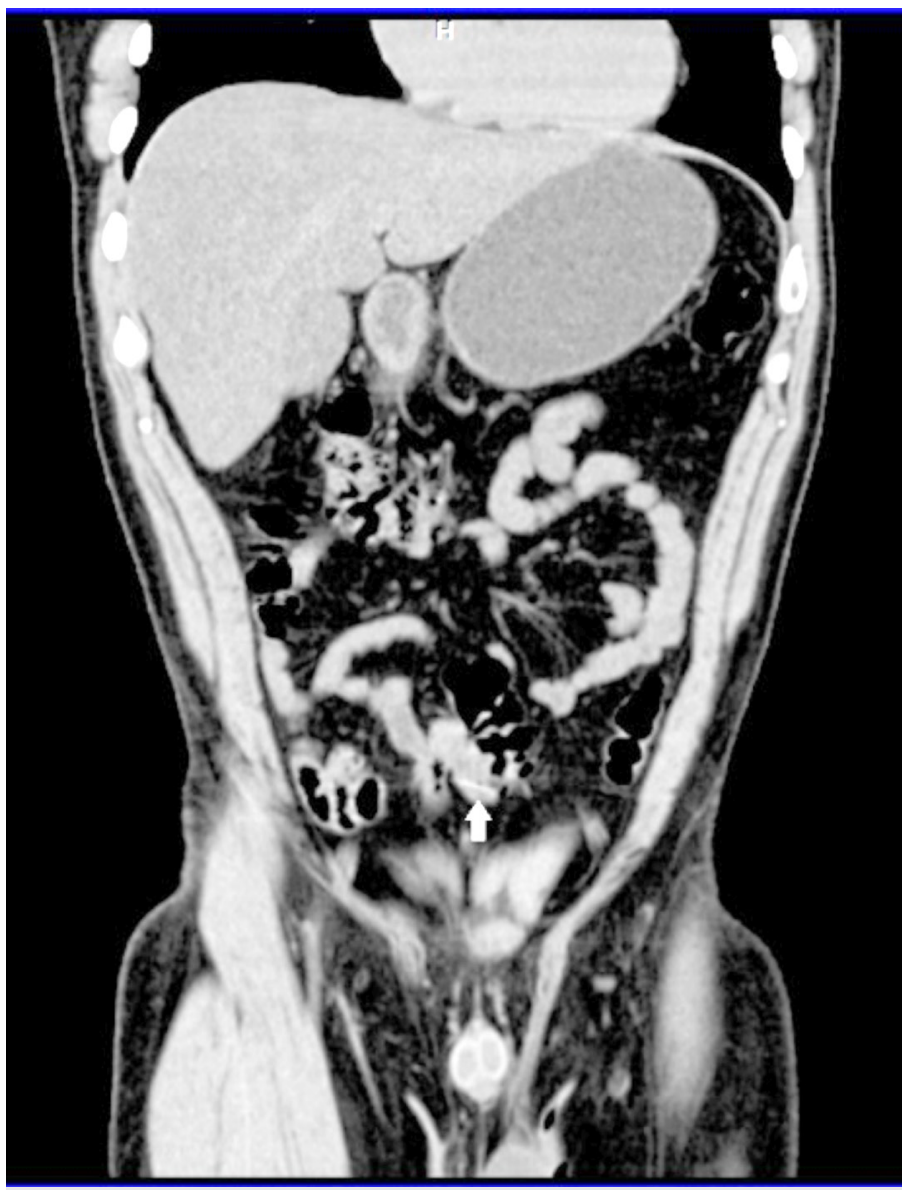

Figura 1. Tomografía que muestra una imagen hiperdensa lineal que atraviesa la pared intestinal del íleon distal. Fuente: autores.

Se estableció el diagnóstico de abdomen agudo secundario a perforación por cuerpo extraño y se decidió manejo quirúrgico mediante abordaje laparoscópico; al no disponer de equipos de laparoscopía se realizó laparotomía infraumbilical.

Se evidenció divertículo de Meckel de $4 \mathrm{~cm}$ de longitud por 1,5 cm de diámetro (índice longitud/diámetro $2,6 \mathrm{~cm}$ ) perforado por espina de pescado en su vértice a $80 \mathrm{~cm}$ de la válvula ileocecal; sin evidencia de plastrón, líquido libre o peritonitis (figura 2).

Se realizó diverticulectomía con auto sutura carga azul $55 \mathrm{~mm}$ en la base, control de hemostasia con puntos simples de poliglactina 3/0 y comprobación de impermeabilidad de la resección mediante prueba de fuga aérea.

La estancia posquirúrgica fue de 48 horas sin complicaciones inmediatas por score de Clavien-Dindo.

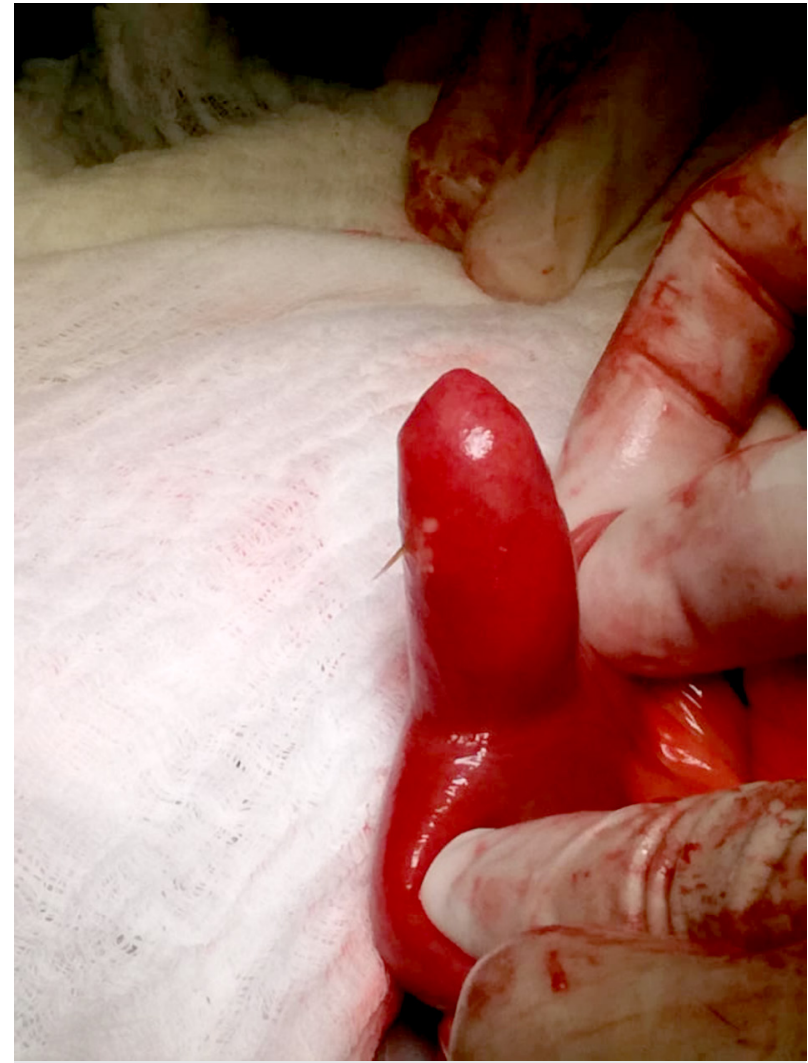

Figura 2. Divertículo de Meckel perforado en tercio distal. Fuente: autores.

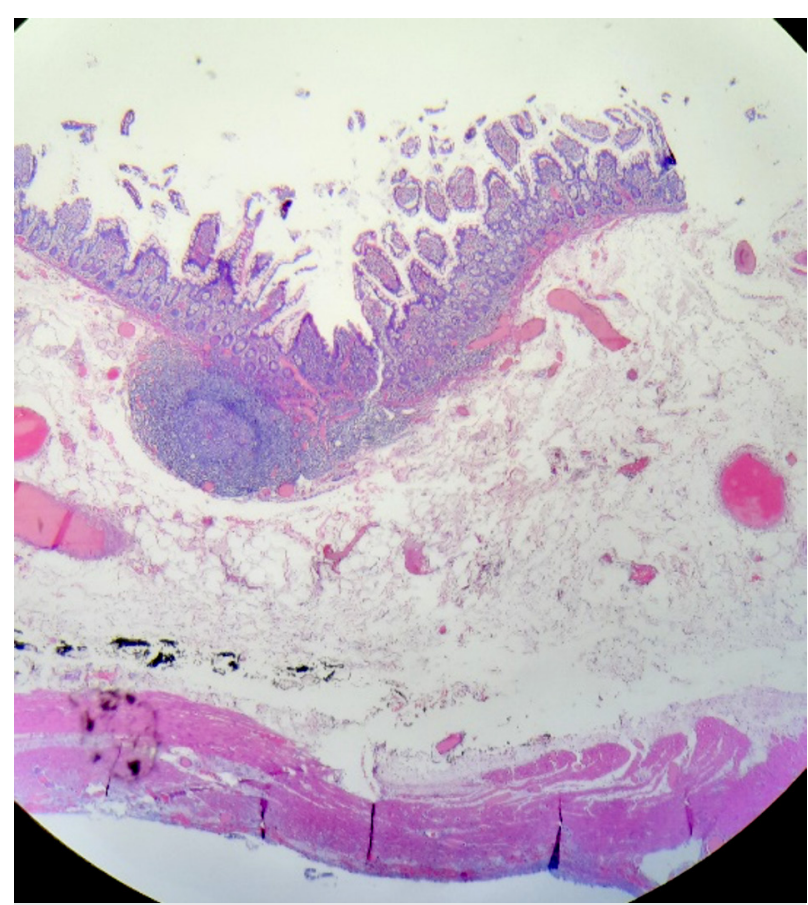

Figura 3. Dilatación sacular revestida por epitelio característico del intestino delgado, epitelio cilíndrico ciliado con vellosidades. Tejido linfoide asociado a la mucosa, tejido adiposo de la submucosa y la serosa. Fuente: autores. 
El departamento de patología reportó tejido diverticular blando que mide $3 \mathrm{~cm}$ por un diámetro de $2,5 \mathrm{~cm}$ en la base y $0,7 \mathrm{~cm}$ en la punta perforada en tercio medio por espina blanquecina verdosa que mide $2,3 \mathrm{~cm}$ con extremo sobresaliente agujado que sobresale del tejido sin evidencia de tejido ectópico (figura 3-4)

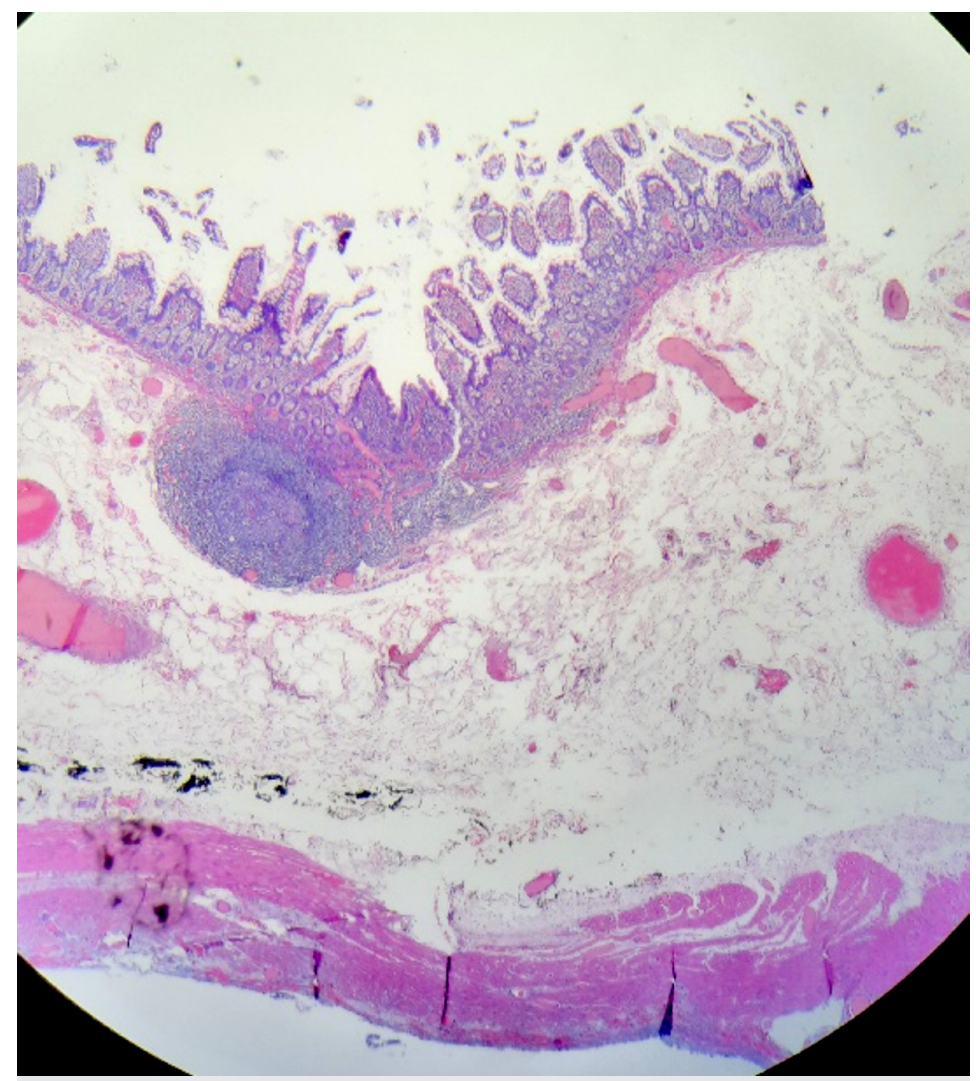

Figura 4. A 400x se observa el tejido conectivo de la subserosa con congestión vascular, hiperplasia mesotelial reactiva e infiltrado inflamatorio linfocitario con escasos polimorfonucleares en relación con proceso inflamatorio crónico agudizado. No se identifica malformación hamartomatosa ni tejido ectópico. Fuente: autores.

Acudió a control al día 13 postquirúrgico sin signos de respuesta inflamatoria sistémica, tránsito intestinal dentro de la normalidad sin evidenciar complicaciones relacionadas al procedimiento quirúrgico (infección del sitio quirúrgico, obstrucción intestinal, hernia incisional).

\section{Discusión}

El divertículo de Meckel es la malformación congénita más común del tracto digestivo; tiene una prevalencia variable de acuerdo a las diferentes publicaciones que va del $0.3 \%-2.9 \%$ en la población general, su incidencia es mayor en el sexo masculino con una relación 1.5-4:1(1-5).

En general, presentan un curso asintomático a lo largo de la vida, pero se estima que el 4,2\% tendrán alguna complicación asociada, y el 3\% requerirán hospitalización y cirugía(7).

Zyluk A. caracteriza al DM por la regla de los "dos": $2 \%$ de la población, niños menores de 2 años de edad, 2 veces más frecuente en hombres y a 2 pies $(60 \mathrm{~cm})$ de distancia desde el ciego(8); además puede estar libre en la cavidad sin conexión con la pared abdominal, unido por un cordón fibroso o como un conducto permeable que resulta en una fistula umbilical en el $74 \%, 24 \%$ y $2 \%$, respectivamente ${ }^{(9)}$.

Su diagnóstico representa un reto, permaneciendo en su gran mayoría no diagnosticada o en su defecto descubierto durante la autopsia. Se pueden emplear rayos $X$, fluoroscopía, ultrasonido, TC, angiografía, endoscopia, resonancia magnética, capsula endoscópica, scan de Meckel (Medicina nuclear-Pertecnetato de tecnecio 99); siendo la laparoscopía diagnóstica la modalidad que lidera de mejor manera a un diagnóstico correcto $^{(3,4,10)}$.

En general tienen un curso asintomático, su presentación clínica es infrecuente y se presentan con obstrucción, hemorragia, inflamación o perforación. Sin embargo, se han reportado presentaciones infrecuentes en la literatura como la presencia de hemoperitoneo espontáneo, intususcepción doble entre otros ${ }^{(9)}$.

Referente a la perforación por cuerpos extraños, esta es considerada una complicación extremadamente rara, existen 6 reportes de perforación del divertículo de Meckel por una espina de pescado a nivel internaciona|(11), cuyo mecanismo fisiopatológico consiste en la entrada accidental al lumen del divertículo de Meckel y posteriormente la peristalsis intestinal lo traslada hacia la punta(12).

Con respecto a los DM asintomáticos (incidentales) es controversial su manejo, según Park et al. el manejo quirúrgico se recomienda basado en el riesgo acumulado del $6,4 \%$ de presentar complicaciones si no son resecados(13).

Además Park et al. recomienda el manejo quirúrgico selectivo ante la presencia de un DM incidental, basándose en factores de riesgo predictores de complicaciones:

1) edad menor de 50 años 2) género masculino 3) divertículo de longitud mayor de $2 \mathrm{~cm}$ y 4) anormalidades macroscópicas sugiriendo la presencia de tejido gástrico ectópico así como un cuello diverticular estrecho; si estos criterios no se cumplen existe un mínimo riesgo de complicaciones pero puede alcanzar hasta el $70 \%$ si se presentan todos(13). 
El riesgo de malignidad de un DM es del 0.5\%-3.2\%, reportándose hasta 70 veces mayor en esta localización que en otras áreas del intestino delgado; son en su mayoría tumores neuroendocrinos (63,2\%), seguidos de GIST (10,5\%), adenocarcinoma $(5,3 \%)$ y neoplasia epitelial pancreática $(5,3 \%)$ y $15,8 \%$ metástasis secundaria(14).

Zani et al. comparó la no resección versus resección de DM incidentales y demostró que el riesgo de complicaciones es menor en el grupo de no resección $1.3 \%$ versus $5.3 \%$, respectivamente). ${ }^{(15)}$

En el caso de DM complicado, la resección quirúrgica es mandatoria mediante diverticulectomía simple (DS), resección en cuña o resección segmentaria ya sea mediante laparotomía o laparoscopía. La laparoscopía es una herramienta útil y segura; necesita mayor cantidad de estudios y pacientes para considerarlo como Gold estándar; tiene desventajas como la dificultad de realizar la resección con auto sutura; sin embargo, el empleo de laparoscopía asistida por mini laparotomía es una opción segura(1,2)

Blouhos et al. menciona varios factores a tomar en cuenta al momento de decidir la técnica quirúrgica; entre ellos: la integridad de la base del divertículo, el íleon adyacente; la presencia o localización de I tejido ectópico. Un DM con índice longitud diámetro mayor a $2 \mathrm{~cm}$ y tejido ectópico en la punta puede ser removido mediante DS; en contraste Robinson et al. describió que se realiza una DS independientemente del tamaño del divertículo con margen libre de tejido ectópico(16,17).

En el presente caso, el DM tuvo un índice longitud/diámetro de 2,6 cm; sin compromiso de su base, por lo que se realizó una DS sin complicaciones, misma que mantiene relación con la evidencia publicada.

Robinson et al. describió una estancia hospitalaria mayor en pacientes pediátricos intervenidos mediante laparotomía comparada con laparoscopía por un DM sintomático (11,4 días versus 2,6 días)(17), por otra parte Hosn et al. en adultos reportó una estancia hospitalaria para el abordaje laparoscópico entre 3 a 5 días, con un $25,7 \%$ de conversión ${ }^{(18)}$. Sin embargo, el paciente fue egresado en 48 horas sin complicaciones.

A causa de la baja tasa de presentación del DM no se tiene información sobre costos en estancia hospitalaria, abordaje quirúrgico en población adulta.

\section{Conclusión}

La presencia de un divertículo de Meckel complicado debe ser tomado en cuenta en la valoración de un abdomen agudo, siendo de vital importancia para el cirujano tener un alto índice de sospecha con la presentación atípica de dolor en el cuadrante inferior derecho; permitiéndonos así su reconocimiento y tratamiento quirúrgico temprano.

\section{Abreviaturas \\ DM: divertículo de Meckel \\ TC: Tomografía computarizada \\ DS: Diverticulectomía simple}

\section{Referencias}

1. Lindeman R-J, Søreide K. The Many Faces of Meckel's Diverticulum: Update on Management in Incidental and Symptomatic Patients. Curr Gastroenterol Rep. enero de 2020;22(1):3.

2. Lequet J, Menahem B, Alves A, Fohlen A, Mulliri A. Meckel's diverticulum in the adult. J Visc Surg. septiembre de 2017;154(4):253-9.

3. Choi S-Y, Hong SS, Park HJ, Lee HK, Shin HC Choi GC. The many faces of Meckel's diverticulum and its complications. J Med Imaging Radiat Oncol. abril de 2017;61 (2):225-31.

4. Kuru S, Kismet K. Meckel's diverticulum: clinical features, diagnosis and management. Rev Esp Enfermedades Dig. 2018;110(10):72632.

5. Hansen C-C, Søreide K. Systematic review of epidemiology, presentation, and management of Meckels diverticulum in the $21 \mathrm{~s}$ century: Medicine (Baltimore). agosto de 2018:97(35):e12154.

6. Gagnier JJ, Kienle G, Altman DG, Moher D, Sox H, Riley D. The CARE Guidelines: Consensus-based Clinical Case Reporting Guideline Development. Glob Adv Health Med. septiembre de 2013;2(5):38-43.
Colvin RW, Al-Katib S, Ebersole J. Perforated Meckel's Diverticulitis. J Gastrointest Surg. diciembre de 2017;21(12):2126-8.

8. Zyluk A. Management of incidentally disco vered unaffected Meckel's diverticulum - a review. POL PRZEGL CHIR. 2019;91 (6):41-6.

9. Francis A, Kantarovich D, Khoshnam N Alazraki AL, Patel B, Shehata BM. Pediatric Meckel's Diverticulum: Report of 208 Cases and Review of the Literature. Fetal Pediat pathol. el 3 de mayo de 2016;35(3):199-206.

10. Chatterjee A, Harmath C, Vendrami CL, Ham mond NA, Mittal P, Salem R, et al. Reminiscing on Remnants: Imaging of Meckel Diverticulum and Its Complications in Adults. Am J Roentgenol. noviembre de 2017;209(5):W287-96.

11. Daniele L, Elliott D, Wong MS, Free J. Perforation of Meckel's diverticulum by an intact fish bone: a case report and literature review. ANZ J Surg. noviembre de 2017;87(11):E206-7.

12. Masuya R, Okamoto K, Kidogawa H, Kamizono J, leiri S. Rare pediatric case of Meckel diverticulum penetration caused by a fish bone. Pediatr Int. julio de 2019;61 (7):731-3.

13. Park JJ, Wolff BG, Tollefson MK, Walsh EE, Larson DR. Meckel Diverticulum: The Mayo Clinic
Experience With 1476 Patients (1950-2002). Ann Surg. marzo de 2005;241(3):529-33.

14. van Malderen K, Vijayvargiya P, Camilleri $M$, Larson DW, Cima R. Malignancy and Meckel's diverticulum: A systematic literature review and 14-year experience at a tertiary referral center. United Eur Gastroenterol J. 2018;6:739-47.

15. Zani A, Eaton S, Rees CM, Pierro A IncidentaIly Detected Meckel Diverticulum. Ann Surg. 2008;247(2):276-81.

16. Blouhos K, Boulas KA, Tsalis K, Barettas N, Pa raskeva A, Kariotis I, et al. Meckel's Diverticu lum in Adults: Surgical Concerns. Front Surg [Internet]. 2018 [citado el 9 de diciembre de 2020]:5. Disponible en: https://www frontiersin.org/articles/10.3389/fsurg.2018.00055/full

17. Robinson JR, Correa $\mathrm{H}$, Brinkman AS, lii HNL. Optimizing surgical resection of the bleeding Meckel diverticulum in children. J Pediatr Surg. 2017;52(10):1610-5

18. Hosn MA, Lakis M, Faraj W, Khoury G, Diba S. Laparoscopic Approach to Symptomatic Meckel Diverticulum in Adults. J Soc Laparoendosc Surg. 2014;18(4):e2014.00349. 